\title{
Two-color beam generation based on wakefield excitation
}

\author{
S. Bettoni, E. Prat, and S. Reiche \\ Paul Scherrer Institut, Villigen 5232, Switzerland \\ (Received 18 February 2016; published 25 May 2016)
}

\begin{abstract}
Several beam manipulation methods have been studied and experimentally tested to generate two-color photon beams in free electron laser facilities to accommodate the user requests. We propose to use the interaction of the beam with an oscillating longitudinal wakefield source to obtain a suitable electron beam structure. The bunch generates two subpulses with different energies and delayed in time passing through a magnetic chicane after its longitudinal phase space has been modulated by the wakefield source. According to this approach the power of the emitted radiation is not degraded compared to the monochromatic beam, and the setup in the machine is quite simple because the bunch is manipulated only in the high energy section, where it is more rigid. We present the design applied to SwissFEL. We identified the parameters and the corresponding range of tunability of the time and energy separation among the two subbunches.
\end{abstract}

DOI: 10.1103/PhysRevAccelBeams.19.050702

\section{INTRODUCTION}

Free electron laser (FEL) facilities are the highest brightness sources used for scientific applications, opening up atomic imaging at femtosecond down to attosecond time resolution. Several FELs are in operation and their success has triggered the development of more facilities around the world. On the way, the success of this kind of machines new operation modes are studied to accommodate the different user requests. One of the modes is the so-called two-color scheme, where two pulses with different photon energy and adjustable time separation are generated. The dynamics of ultrafast processes can be studied using a highintensity X-ray pump and probe to reach time resolution at the level of a few femtoseconds. This technique has already been successfully applied to numerically study warm dense matter [1,2] as well as multiple wavelength anomalous dispersion imaging [3] using serial femtosecond crystallography [4].

The wavelength of the radiation emitted in a FEL, $\lambda_{R}$, may be expressed as a function of the $K$ parameter of the undulators as [5]:

$$
\lambda_{R}=\frac{\lambda_{U}}{2 \gamma^{2}}\left(1+\frac{K^{2}}{2}\right)
$$

where $\lambda_{U}$ is the undulator period, and $\gamma$ the beam Lorentz factor.

\footnotetext{
*simona.bettoni@psi.ch

Published by the American Physical Society under the terms of the Creative Commons Attribution 3.0 License. Further distribution of this work must maintain attribution to the author $(s)$ and the published article's title, journal citation, and DOI.
}

The first technique used to generate the two colors is based on the dependence of the radiation wavelength on the undulator field parameter. The two colors are generated using distinct values of $K$ with a quasi monoenergetic electron beam [6,7]. This method has been proved experimentally at the Linac coherent light source (LCLS). The main advantages of this technique are the large tunability of the time delay (from 0 ps up to few hundreds of fs), the energy separation (up to few tens of percent), and the synchronization of the two photon pulses, albeit the total power is typically strongly reduced (about 1/10 of the full saturation power for the LCLS case [6]).

Another scheme, called twin-bunch approach, has been proposed and experimentally demonstrated at optical wavelengths [8] and in the range of X-rays [9]. This method makes use of the pulse-stacking technique, used in several photo-injectors to obtain a longitudinal flat laser profile $[10,11]$. Besides generating two-color FELs, the twinbunch scheme can also find application in beam or wakefield driven plasma acceleration. Compared with the masking technique adopted now [12], this method gives more flexibility in the control of the charge distribution, peak current, and time delay among the two pulses.

In contrast to the previous approach based on distinct values of $K$, in the twin-bunch scheme each subpulse of the electron beam efficiently participates to the lasing process, producing a larger X-ray power, albeit the tunability of the time and energy separation of the two-pulses is limited and the setup of the machine requires a longer procedure compared to the $K$ method. In this case the time and the energy separation among the two subpulses is smaller than in the previous method, and they are not independent. A discussion about how to control these quantities in the twinbunch approach has been presented in [13]. Some knobs 
have been identified to be used alone or in combination to vary the time and the energy separation of the subpulses, but some correlation among these two quantities is still present and a current variation of the subpulses has to be tolerated. The latter limitation arises from the fact that the beam dynamics is very different for the two subpulses mode and the nominal operation setup. Consequently in the twin-bunch mode the machine has to be tuned in the low energy section (energy smaller than about $10 \mathrm{MeV}$ ).

We propose to generate two electron pulses separated in time and energy using the interaction of a longitudinal wakefield excited by the beam passing through a dielectriclined or a corrugated waveguide. In the last years several possibilities have been considered to manipulate a relativistic bunch using its interaction with the wakefield excited by the beam passing through this kind of structure. They include the possibility to control the energy chirp [14,15], linearize the longitudinal phase space beam distribution residual from the bunch compression process [16,17], drive coherent terahertz radiation [18,19], and do longitudinal diagnostics [20]. We propose to use the longitudinal wakefield to super-impose to the global chirp (obtained by going-off-crest in the rf structures) modulations at two locations along the beam longitudinal phase space. Properly choosing the wavelength of the wakefield we can generate energy chirps of opposite sign along different intervals of the longitudinal coordinate of the beam. The bunch with such a modulation passing through a magnetic chicane is then compressed, generating the two-color modes. In this case the low energy section of the machine is not modified compared to the nominal setup, and by this our proposed approach eases the setup compared to the twin-bunch scheme. Some knobs have been identified to control the time and energy separation of the two subpulses in [13], accepting a variation of the peak current of the subpulses and some dependence of the time delay on the energy separation. In our scheme we could in principle use the same knobs since the beam structure at the exit of the low energy section is similar to the one of the twin-bunches case. We also identified two knobs to independently control the time and energy separation of the two subpulses and at the same time keeping constant the peak currents of the two subpulses.

Other methods have been studied and experimentally tested to generate subpulses separated in time and energy using the hardware dedicated to the seeding schemes.
Different wavelengths of the seeding laser have been used $[21,22]$, or the crystal used in the self-seeding scheme has been oriented to allow for two reflections, producing different wavelengths in the transmitted X-ray pulses [23].

We applied and optimized our method to SwissFEL, the FEL facility briefly described in Sec. II. We will present the scheme in Sec. III, identifying a suitable source of the wakefield in terms of wavelength and amplitude (subsection III A), and we will show a possible geometry (subsection III B). Finally we will discuss the tunability of time delay and energy separation among the subpulses (subsection III C).

\section{THE SwisSFEL FREE ELECTRON LASER FACILITY}

SwissFEL [24] is the FEL facility under construction at the Paul Scherrer Institute (PSI). Its hard and soft X-ray beam lines, Athos and Aramis, will produce photon pulses with wavelengths ranging from 0.1 to $0.7 \mathrm{~nm}$, and from 0.7 to $7 \mathrm{~nm}$, respectively. The commissioning of the accelerator is foreseen to start in fall 2016 and the first pilot user experiments are expected before the end of 2017. The schematic layout of the machine is shown in Fig. 1. The electron beam emitted from a cesium-telluride photocathode illuminated by a UV-laser will be accelerated up to $150 \mathrm{MeV}$ by two S-band cavities, preceding a laser heater system used to mitigate the microbunching instability [25]. The beam energy will then be boosted to $330 \mathrm{MeV}$ by other S-band cavities, before the bunch will be compressed in the first bunch compressor (BC1). The beam will then be accelerated to $2.1 \mathrm{GeV}$ by $\mathrm{C}$-band cavities before being compressed further in the second bunch compressor (BC2) to reach the short bunch lengths necessary for the lasing in the X-ray region. The most relevant parameters for the two compression stages at SwissFEL are listed in Table I for the maximum bunch charges foreseen.

The S-band cavities between the laser heater and $\mathrm{BC} 1$ and the C-band structures between the two bunch compressors will be run at off-crest phase to generate the energy-time correlation for the compression with a phase $\phi_{1}$ and $\phi_{2}$, respectively. The C-band cavities downstream of $\mathrm{BC} 2$ will be run on-crest in normal operation at phase $\phi_{3}$, since these cavities are mainly used to increase the beam energy up to $5.8 \mathrm{GeV}$ at the entrance of the Aramis line and their longitudinal wakefield will reduce the residual relative

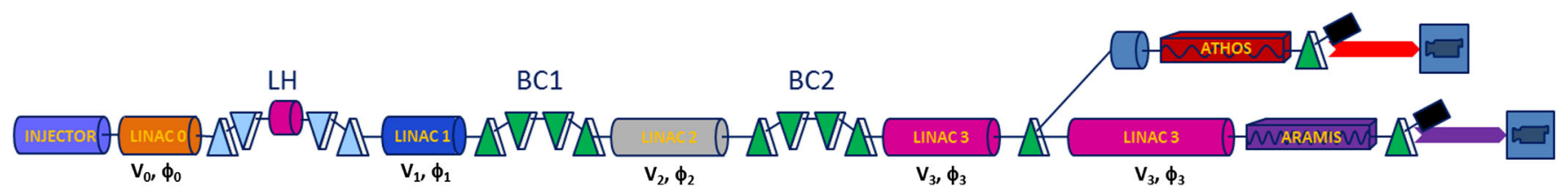

FIG. 1. SwissFEL schematic layout. The symbols for the voltages $V_{i}$ and the phases $\phi_{i}$ along the different sections in the machine used in the discussion are also indicated. 
TABLE I. Beam parameters at the $\mathrm{BC} 1$ and $\mathrm{BC} 2$ exit, and at the entrance of the Aramis undulator line in SwissFEL. The bunch charge is $200 \mathrm{pC}$, the laser length $10 \mathrm{ps} \mathrm{FWHM,} \mathrm{and} \mathrm{the} \mathrm{peak}$ current at the entrance of $\mathrm{BC} 1$ is $20 \mathrm{~A}$.

\begin{tabular}{lccc}
\hline \hline & $\mathrm{BC} 1$ & $\mathrm{BC} 2$ & Aramis \\
\hline Peak current $(\mathrm{kA})$ & 0.233 & 3 & 3 \\
$R_{56}(\mathrm{~mm})$ & 55.1 & 20.7 & 0 \\
Beam energy $(\mathrm{GeV})$ & 0.33 & 2.10 & 5.8 \\
\hline \hline
\end{tabular}

energy chirp from the compression process cumulated in the previous stages.

\section{LONGITUDINAL PHASE SPACE MANIPULATION}

In the previous schemes used to generate the two-color operation mode the beam/radiation properties were modified at the end of the machine in the undulator lines [6] or at the location of the electrons emission from the photocathode [9]. These methods present both advantages and drawbacks. The first approach provides full control of the time and energy separation of the two subpulses, but implies a reduction of the total emitted power with respect to the case of the quasi monoenergetic configuration. The twin-bunch scheme overcomes this limitation, but it introduces other issues, such as the difficulty in the setup, and a study has shown that there is a correlation between the time and the energy separation of the sub-bunches [13].

We propose to modulate the beam longitudinal phase space upstream of $\mathrm{BC} 1$ using the longitudinal oscillating wakefield excited by the bunch itself passing through an opportune device, and to use the same compressor to generate the current peaks. In this case each current peak lases independently and reaches saturation generating the maximum FEL power, as for the twin-bunch scheme. On top of this, the bunch is not modified in the low energy section of the machine, so that no degradation is expected and the setup is easier than in the case of the twin-bunches case. Since the beam longitudinal phase space structure obtained with our method is very similar to the twin-bunch configuration, we could in principle use the knobs identified in [13] to tune the time and energy separation of the subpulses, accepting a reasonable peak current variation. To these knobs we preferred to use the tunable wavelength of the wakefield and the phase $\phi_{3}$. These parameters allows to independently control the time and the energy separation of the two subpulses keeping a constant value of the peak currents. A detailed description of the tunability of the system will be given in Sec. III C.

Our scheme requires an oscillating wakefield source with a characteristic wavelength $\lambda_{W}$, which satisfies the condition:

$$
\lambda_{W} \approx L_{z} / 2
$$

where $L_{z}$ is the full bunch length of the uncompressed beam. In this way we can generate chirps with different signs along the longitudinal coordinate of the bunch, $s$. As an example Fig. 2 shows the comparison between the SwissFEL nominal $200 \mathrm{pC}$ charge beam longitudinal phase space and that corresponding to a bunch passing through a wakefield source satisfying the condition in Eq. (2). A chirp is applied as in the nominal operation mode by going offcrest in the structures upstream of $\mathrm{BC} 1$ and on top of that the sinusoidal wake potential is used to generate the two chirps. Such a beam passing through $\mathrm{BC} 1$ will produce the two current peaks along the bunch.

By changing the ratio of $\lambda_{W}$ over $L_{z}$ the two-color scheme may easily become a setup for a multicolor operation mode, with a limited tunability compared to the two-color case (time and energy separation may not be changed individually for the subpulses).

\section{A. Longitudinal wakefield source}

Several longitudinal wakefield sources are suitable to generate a pattern as the one shown in Fig. 2. Among the different possibilities we consider the cases of a corrugated plate and a dielectric tube, because of their large wakefield amplitude (typically of the order of $10^{16}-10^{18} \mathrm{~V} / \mathrm{C} / \mathrm{m}$ ) and the wavelength range (from hundreds of microns to millimeters assuming reasonable geometries from a
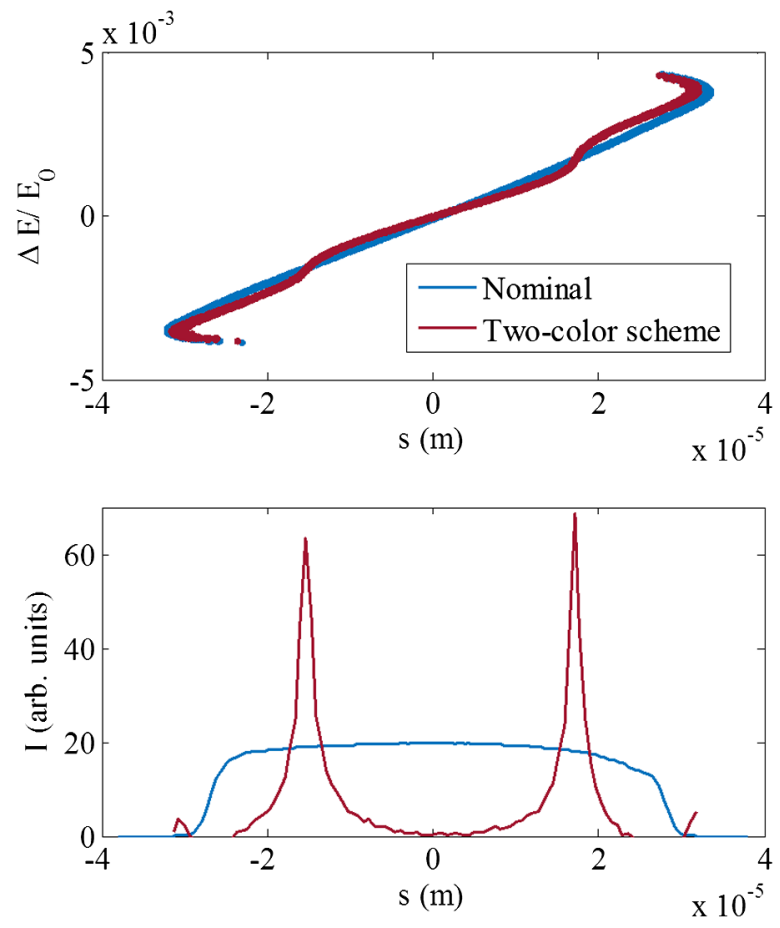

FIG. 2. Comparison of the beam longitudinal phase space at the entrance of $\mathrm{BC} 1$ (top) and current longitudinal profile at the entrance of $\mathrm{BC} 2$ (bottom) corresponding to the nominal operation mode of SwissFEL and the one assuming an opportune wakefield source. 
manufacturing point of view). In the case of the corrugated plate an adjustable gap may be used to tune the wakefield amplitude and wavelength, albeit the strength is reduced by a geometrical factor of $\pi^{2} / 16$ compared to the cylindrical case assuming the same aperture [26]. The intensity of the wakefield of a corrugated plate is enough to shape the beam longitudinal phase space assuming a reasonable length (below $2 \mathrm{~m}$ ) considering the SwissFEL beam energy and $R_{56}$ at the first compressor. We therefore chose the planar geometry, because of its better flexibility to control the wavelength in despite of the smaller intensity of the wakefield compared to the cylindrical case. The optimization procedure and the considerations are general, and therefore valid also for other wakefield sources characterized by the same wavelength and strength.

Figure 3 shows the schematic layout of a corrugated plate.

The longitudinal point-charge wake function, $w(s)$, is given by [27]:

$$
w(s) \sim \frac{\pi}{16} \frac{Z_{0} c}{\pi a^{2}} H(s) \cos \left(\frac{2 \pi}{\lambda_{W}} s\right),
$$

where $Z_{0}$ and $c$ are the free space impedance and the vacuum speed of light, respectively, and $H(s)$ is the unit step function. The other parameters depend on the geometry of the corrugation and the semiaperture $a$. The expression in Eq. (3) is valid only under the following assumptions with reference to Fig. 3: (i) $\delta$ and $p \ll a$, (ii) $\delta \geq p$. If these conditions are satisfied the wake potential has a sinusoidal dependence on $s$ with wavelength given by:

$$
\lambda_{W}=2 \pi \sqrt{\frac{a \delta g}{2 p}} .
$$

We see that the wavelength of the wake potential depends on the geometry of the plate and the semigap, whereas the

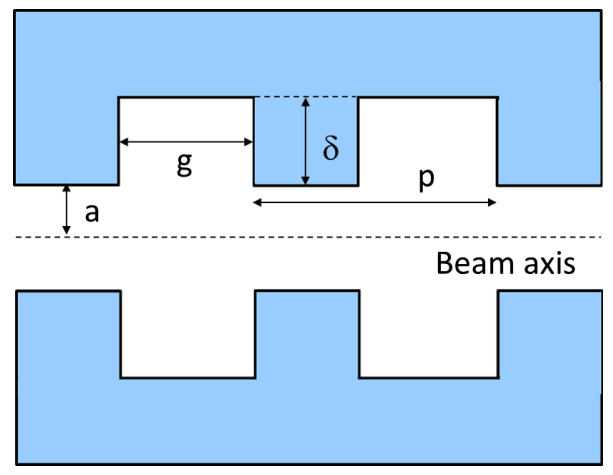

FIG. 3. Section of the geometry of a corrugated plate. The most important parameters for the wakefield calculations are also indicated. amplitude is only a function of $a$. We used these considerations to dimension our system for SwissFEL.

\section{B. Application to SwissFEL}

The optimal location to install the corrugated plate is upstream of the first bunch compressor to maximize the relative energy chirp and minimize the impact on the machine at lower energies compared to the nominal setup. At SwissFEL there is $8 \mathrm{~m}$ of available space where the corrugated plate may be accommodated between the last S-band structure and the entrance of $\mathrm{BC} 1$. The most important beam parameters at this location are shown in Table II.

We optimized the geometry and the length of the corrugated plate using the numerical codes Elegant [28] and LiTrack [29]. The first code determines the particles distribution at any position along the accelerator applying matrix transformations to the initial beam $6 \mathrm{D}$ phase-space. It convolutes the wake function with the beam distribution, and computes space charge forces, albeit only along the longitudinal dimension. LiTrack is a faster code, which includes the longitudinal dynamics neglecting any space charge force both in the transverse and in the longitudinal dimension. Considering the relatively low beam charge density and high energy at the location where the plate may be installed we used the latter code to perform all the optimizations, as the space charge forces are negligible. We verified with Elegant the tracking of some reference cases and we used these distributions to obtain information on the power of the emitted radiation using Genesis [30]. We assumed a minimum semiaperture of the plate, $a$, of $1 \mathrm{~mm}$. Since the transverse rms beam size at the location where the corrugated plate may be installed is smaller than $100 \mu \mathrm{m}$ (see Table II), this seemed to be a conservative assumption.

The induced relative energy chirp is proportional to the length of the corrugated plate, $L_{P}$, and the inverse of the square of the semiaperture $a$, therefore any combination of these quantities keeping constant the ratio $L_{P} / a^{2}$ is equivalent from the beam dynamics point of view. We tuned the length of the corrugated plate to have a factor 3 between the saturation length at the slices along the beam corresponding to the current peaks and those at the low current regions assuming $a$ equal to $2 \mathrm{~mm}$. In this way we have some margin to reduce $a$ going towards smaller $\lambda_{W}$, while keeping $L_{P}$ below $2 \mathrm{~m}$. Figure 4 shows the power

TABLE II. Beam parameters at the location where the corrugated plate could be installed at SwissFEL.

\begin{tabular}{lr}
\hline \hline Parameter & Value \\
\hline Beam energy $(\mathrm{MeV})$ & 330 \\
Beam length FWHM $(\mathrm{mm})$ & 3 \\
Beam rms size $\sigma(\mu \mathrm{m})$ & $40<\sigma<80$ \\
Peak current $(\mathrm{A})$ & 20 \\
\hline \hline
\end{tabular}



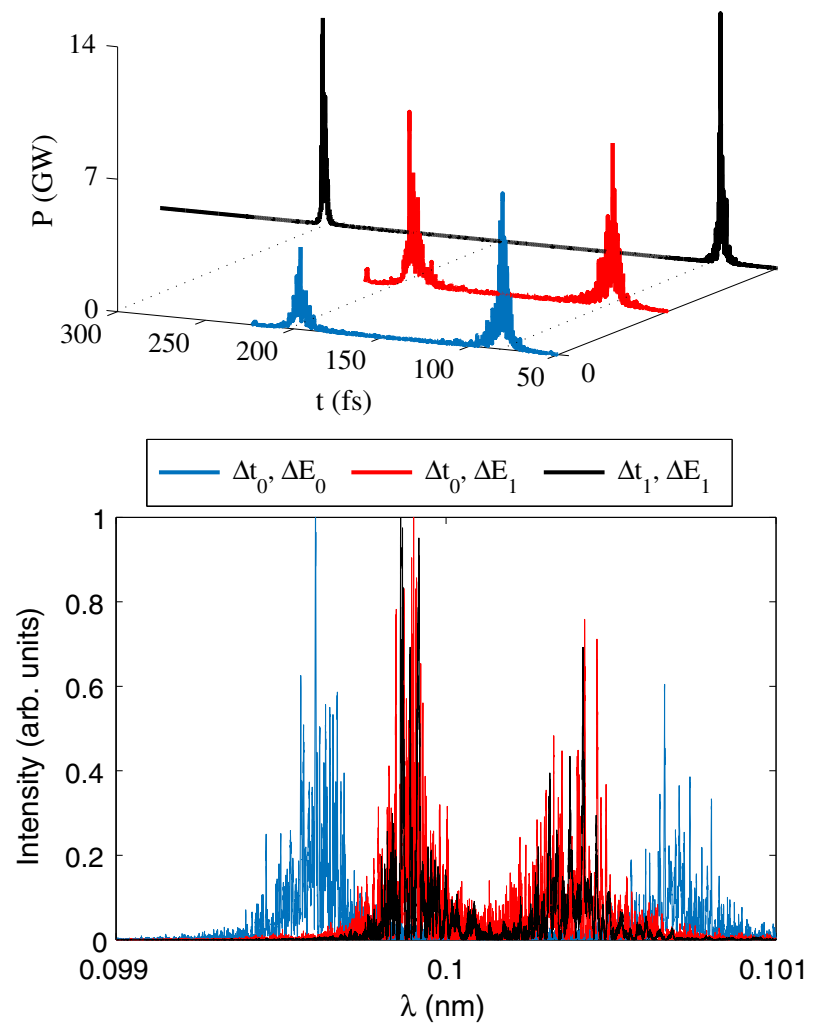

FIG. 4. Power of the emitted radiation along the longitudinal coordinate of the beams (top) and corresponding spectra (bottom). The subscript 0 refers to the reference case. Results corresponding to configurations with a different time delay $\Delta t_{1}$, and energy separation $\Delta E_{1}$ obtained changing the semigap $a$ of the corrugated plate and the phase of the accelerating cavities downstream of $\mathrm{BC} 2$, respectively, are also shown. More discussion can be found in subsection III C.

along the electron beam longitudinal coordinate and the spectrum of the emitted radiation at the end of the Aramis line simulated using Genesis for cases corresponding to different time delays and energy separations of the subpulses. As it can be seen in the top plot of Fig. 4 the radiation power generated by the slices of the beam corresponding to the current spikes is much larger than that produced by the slices in between the spikes.

According to Eq. (4) the wavelength of the wakefield is function of only the three geometrical parameters $p, g$ and $\delta$ and the semiaperture of the plate $a$. A variation of any of them is equivalent to a change of one of the others from the beam dynamics point of view. Figure 5 shows the dependence of $\lambda_{W}$ on $a$ and $\delta$ assuming a $g$ over $p$ ratio equal to $1 / 3$. We selected the geometries characterized by a $g / p=1 / 3$, as this is a good compromise between the simplicity of the manufacture and the range of the achievable wavelengths. We finally selected the values of $g$ and $p$ to satisfy the conditions of validity of the wakefield expression in Eq. (3). The parameters chosen for our design are summarized in Table III. This is only one of the possible geometries, chosen in our case more or less

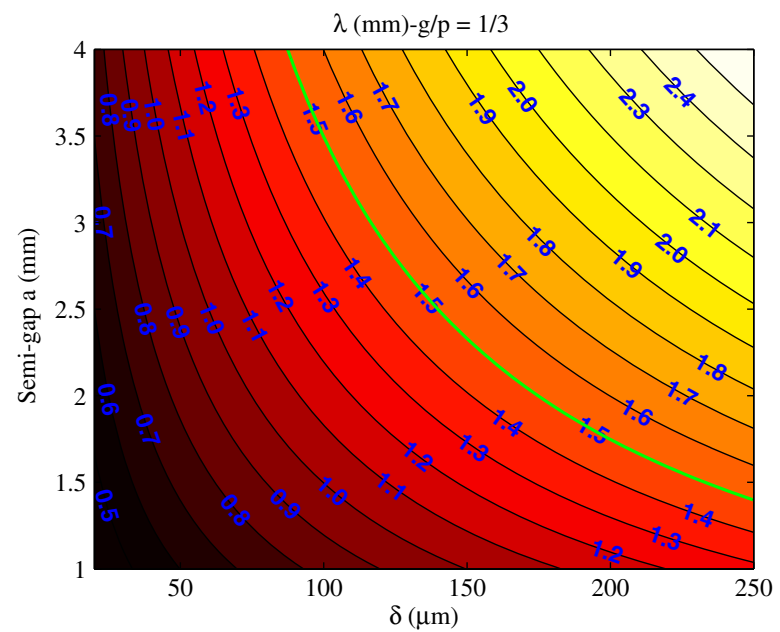

FIG. 5. Wavelength of the wakefield generated by a corrugated plate as a function of $\delta$ and $a$ assuming a ratio $g$ over $p$ equal to $1 / 3$. The level corresponding to the case $\lambda_{W}$ equal to half of the total bunch length is also highlighted.

arbitrarily from manufacturing considerations. In the following discussion we will consider this case as the reference geometry and we will indicate its parameters with the 0 subscript.

To have an idea of the orbit jitter tolerance, we computed the centroid position along the bunch assuming different beam-structure offsets. A $50 \mu \mathrm{m}$ transverse offset at the corrugated plate translates to a difference of the centroid position of the slices corresponding to the peak currents of about $3 \mu \mathrm{m} \mathrm{rms}$, which gives less than $10 \%$ energy degradation. This is an overestimation of the beam degradation, because we consider the case that all the slices lasing have the maximum offset of the centroid position. The expected maximum orbit jitter at the location of the corrugated plate is smaller than $10 \mu \mathrm{m}(1 / 10$ of the rms size of the beam at that location), so this does not seem to be critical for the system.

In the simulations we neglected the quadrupolar component of the wakefield. This will give a focusing along the different slices of the bunch. We consider this to be compensated by using an even number of modules, as done at LCLS for parameters more critical than ours (longer structures and smaller semigap with double beam energy) [31].

TABLE III. Parameters of the geometry of the corrugated plate optimized for SwissFEL.

\begin{tabular}{lr}
\hline \hline Parameter & Value \\
\hline$g(\mu \mathrm{m})$ & 25 \\
$p(\mu \mathrm{m})$ & 75 \\
$\delta(\mu \mathrm{m})$ & 170 \\
Minimum $a(\mathrm{~mm})$ & 1 \\
$a$ corresponding to $\lambda_{W}=1.5 \mathrm{~mm}\left(L_{z} / 2\right)(\mathrm{mm})$ & 2 \\
$L_{P}(\mathrm{~m})$ & 1.5 \\
\hline \hline
\end{tabular}




\section{Tunability}

An important request for the two-color operation mode is the possibility of tuning independently the time delay and the energy separation among the subpulses. In the case of the twin-bunch scheme several quantities have been identified to modify the time and the energy separation [13]. According to this scheme the time delay among the two bunches may be increased by reducing the beam energy at the second bunch compressor, increasing the amplitude of the wakes acting on the bunch, the beam charge, the value of $R_{56}$ of the second bunch compressor, or changing simultaneously the phases of the cavities upstream the first bunch compressor and in between the two compressors. Since the beam longitudinal phase space distribution obtained applying our scheme is quite similar to the twin-bunch case, the same knobs are expected to be usable for the tuning of the device. Figure 6 shows as an example the time and the energy separation among the two subpulses at the entrance of the Aramis undulator line as a
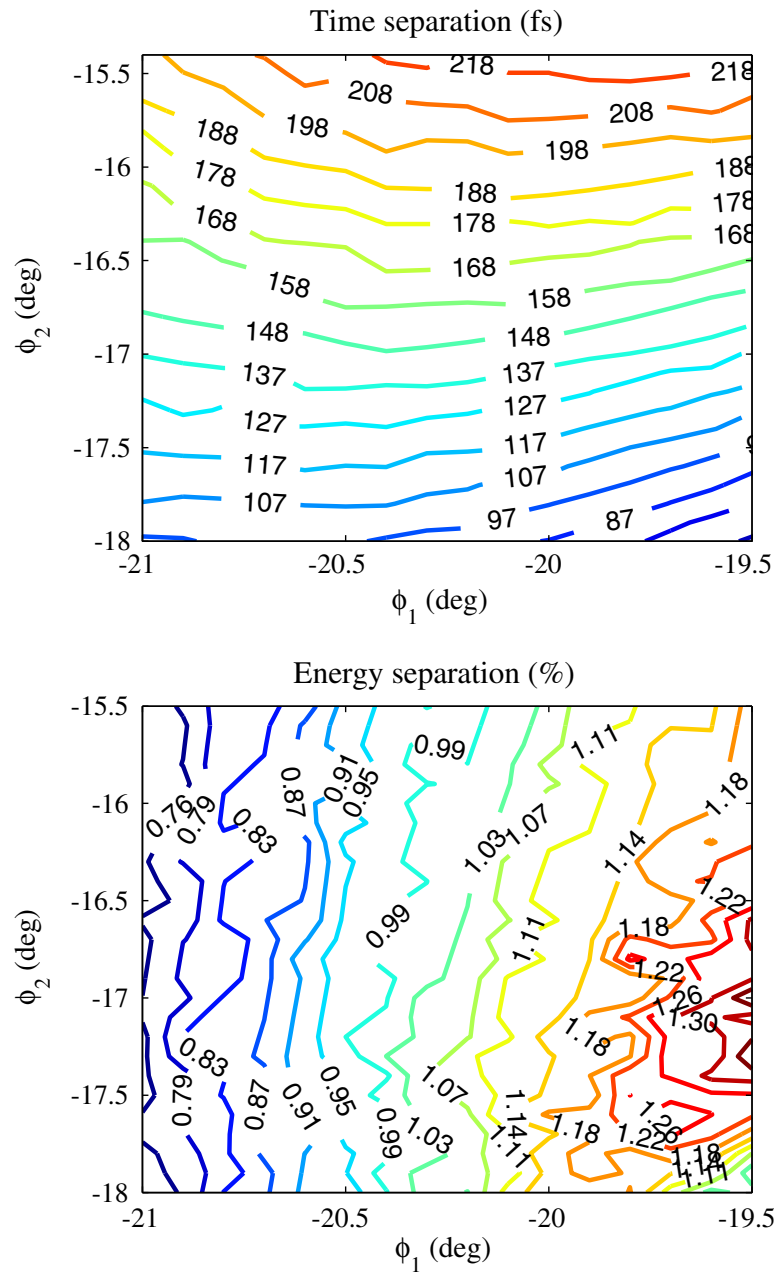

FIG. 6. Time (top plot) and energy (bottom plot) separation among the subpulses assuming different phases upstream of $\mathrm{BC} 1$ $\left(\phi_{1}\right)$ and between $\mathrm{BC} 1$ and $\mathrm{BC} 2\left(\phi_{2}\right)$. function of the phases $\phi_{1}$ and $\phi_{2}$. Similarly to the twinbunch scheme, the time and the energy separation may be controlled almost independently by appropriately tuning one knob at a time or a combination of them, but a certain variation of the peak currents has to be accepted (in the case corresponding to Fig. 6 the current peaks are between $2 \mathrm{kA}$ and $10 \mathrm{kA}$ ). As already shown in [13] if the peak current is constrained in a certain range there is a correlation between the time and energy separation between the subpulses for configurations with large time delay and large energy separation.

In our scheme we may use the dependence of $\lambda_{W}$ on $a$ [see Eq. (4)] to vary the time delay among the subpulses. According to Eq. (3) the amplitude of the wakefield depends on the semiaperture of the corrugated structure, therefore for each value of $a$ there is a unique length of the device which produces the same effect on the beam. This length can be expressed as:

$$
L_{P}\left(\lambda_{W}\right)=\frac{L_{0}}{a_{0}^{2} k_{g}^{4}} \lambda_{W}^{4}
$$

where $\lambda_{W}$ is a generic wavelength of the wakefield, which may be written as:

$$
\lambda_{W}=k_{g} \sqrt{a},
$$

the 0 subscript corresponds to the reference geometry ( $a_{0}=2 \mathrm{~mm}$, and $L_{0}=1.5 \mathrm{~m}$ ), and $k_{g}$ depends on the geometry:

$$
k_{g}=2 \pi \sqrt{\frac{\delta g}{2 p}} .
$$

Figure 7 shows this length and the wavelength of the wakefield as a function of the semiaperture of the

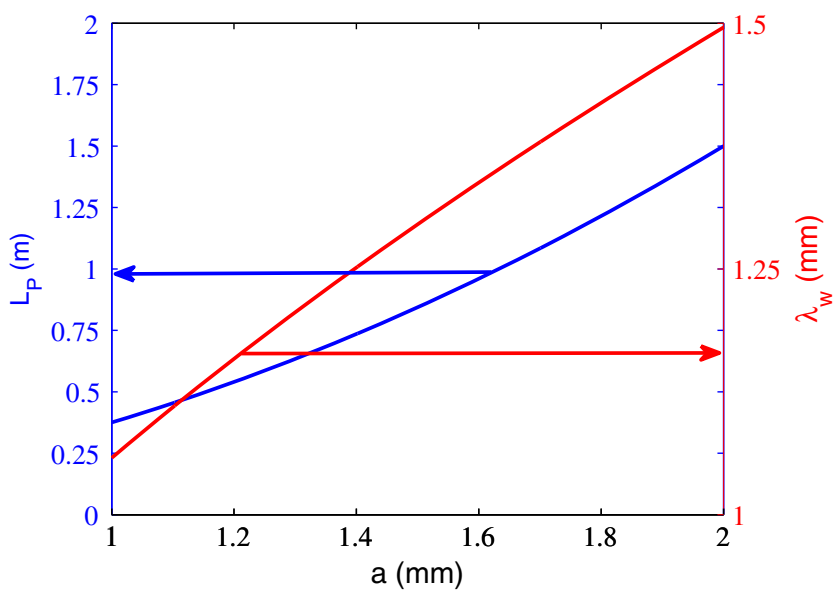

FIG. 7. Length of the corrugated plate equivalent to the reference case ( $a=2 \mathrm{~mm}, L_{P}=1.5 \mathrm{~m}$ ) as a function of $a$ (left axis) and wavelength of the corrugated plate as a function of the semiaperture (right axis). 


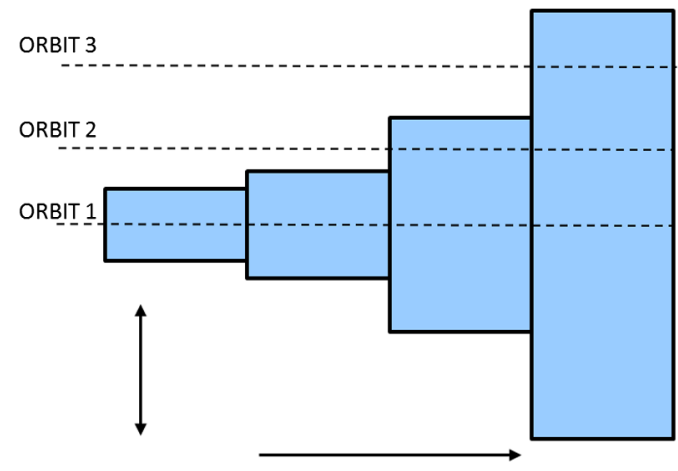

FIG. 8. Top view of the corrugated plate. Electron beams with different offsets see different lengths of the device.

corrugated plate. If the length of the device is chosen as shown in Fig. 7 the peak current of the sub-bunches is the same as for the case corresponding to our reference geometry without changing any other knob in the machine.

The beam passing through a corrugated plate excites not only the dipolar mode considered until here, but also a quadrupolar component because of the broken symmetry of the system around the beam axis. Pairs of corrugated plates rotated by 90 degrees are normally used to compensate for this effect [31]. Corrugated plates of different lengths may be used to discretely compensate for the wakefield amplitude variation with $a$, as schematically shown in Fig. 8. In this way passing with an off-axis trajectory or equivalently moving the plates transversally to the beam longitudinal trajectory may be possible to control the length of the corrugated plate experienced by the beam.

Another possible way to vary the wavelength without affecting the amplitude of the wakefield is to modulate the plate putting a depth of corrugation changing along the transverse dimension. In this way, shifting the structure or the beam orbit, the distance of the subpulses may be modified keeping constant the peak current.

The possibility of changing the length of the corrugated plate adds flexibility to our system. In case we chose the length of the device for each value of $a$ equal to the length $L_{P}$ we may reduce the time separation among the subpulses by closing the gap of the corrugated plate. On the contrary we may increase the time separation among the pulses closing the gap of the device using a length of the device larger than $L_{P}$. In this case, in fact, we have to reduce the global chirp generated by the rf structures to keep a constant peak current of the subpulses, and, by this, we put away the subpulses in time. As an example Fig. 9 shows the current profile obtained assuming $a$ of $1 \mathrm{~mm}$ and a plate length of $1 \mathrm{~m}$, instead of about $0.4 \mathrm{~m}$ corresponding to the $L_{P}$ computed from Eq. (5) for this semigap. In this case we can increase the time separation by $50 \%$ compared to the reference configuration. Furthermore if we prevent the central part of the bunch from lasing, for example using a foil in a dispersive section to degrade the emittance only
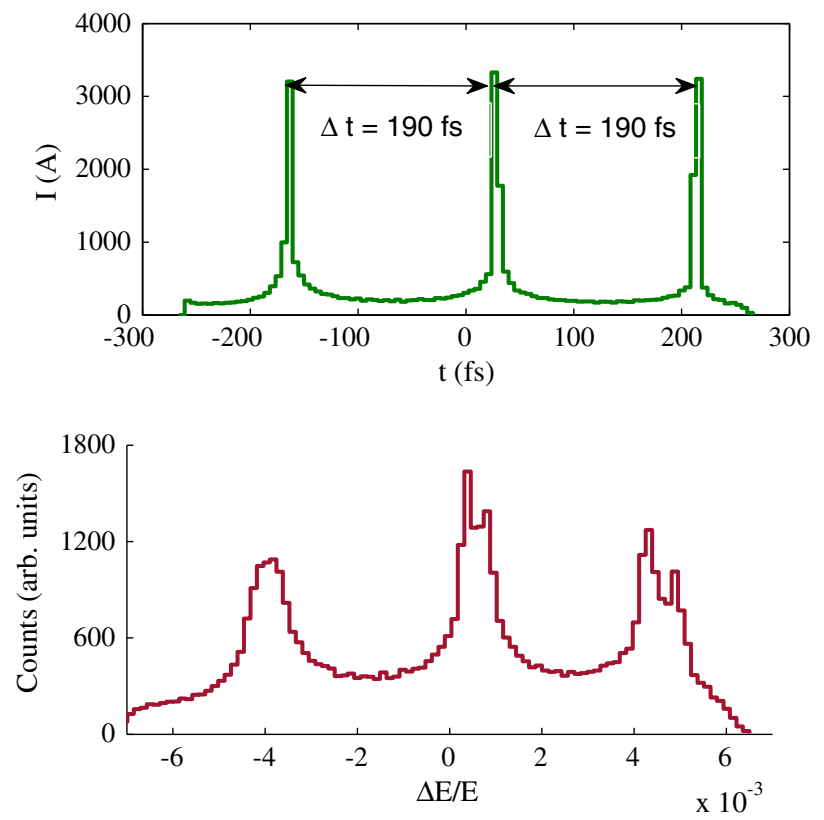

FIG. 9. Current profile corresponding to the configuration $a=1 \mathrm{~mm}, L_{P}=1 \mathrm{~m}$.

in the central section of the beam in the longitudinal dimension [32], we could further double the time separation among the subpulses, using the first and the last subpulse. In this case we can reach a time separation among the pulses of about $370 \mathrm{fs}$. This configuration may also be used to obtain a three-color operation mode.

We identified $\phi_{3}$ as the knob we may use to modify the energy separation among the subpulses without changing the current peak of the sub-bunches. In nominal operation these cavities will be run on-crest to boost the beam energy from 2.1 to $5.8 \mathrm{GeV}$ and their wakefields will be used to compensate the residual energy chirp from the compression process. In the best scenario, i.e., in the range of $\phi_{3}$ between -90 and +90 degrees with respect to the on-crest phase, we expect that the energy separation among the two subpulses, $\Delta E$, ranges from $0.6 \%$ to $1.5 \%$. Limiting the variation of $\phi_{3}$ to \pm 60 degrees, which corresponds to the maximum beam energy variation we can compensate increasing the amplitude of the cavities downstream of $\mathrm{BC} 2$, we expect $\Delta E$ to be from about $0.6 \%$ up to more than $1.1 \%$. Considering that $R_{56}$ downstream of $\mathrm{BC} 2$ is zero, the current peaks of the sub-bunches are the same as for the reference geometry configuration in all the cases.

Table IV summarizes the ranges of the time and energy separation expected for the SwissFEL case keeping the same peak current of both subpulses. In Table IV we report the cases where the global chirp introduced going off-crest in the rf structures corresponds to the under compression layout (positive). In this configuration the high energy subpulse arrives before the low energy one. Setting the phases of the rf cavities to reverse the sign of the chirp (over 
TABLE IV. Time and energy separation of the sub-pulses assuming different values of $a$ and phase of the cavities downstream of $\mathrm{BC} 2$. In the case of $90^{\circ}$ the two subpulses are not accelerated.

\begin{tabular}{lrcc}
\hline \hline & $\begin{array}{r}\Delta t \\
(\mathrm{fs})\end{array}$ & $\begin{array}{c}\Delta E_{\min } \\
(\%)\end{array}$ & $\begin{array}{c}\Delta E_{\max } \\
(\%)\end{array}$ \\
\hline$a=1 \mathrm{~mm}, \Delta \phi_{3}= \pm 60^{\circ}$ & 84 & 0.61 & 0.92 \\
$a=2 \mathrm{~mm}, \Delta \phi_{3}= \pm 60^{\circ}$ & 125 & 0.62 & 1.14 \\
$a=1 \mathrm{~mm}, \Delta \phi_{3}= \pm 90^{\circ}$ & 84 & 0.59 & 1.20 \\
$a=2 \mathrm{~mm}, \Delta \phi_{3}= \pm 90^{\circ}$ & 125 & 0.48 & 1.63 \\
$a=1 \mathrm{~mm}, \Delta \phi_{3}= \pm 60^{\circ}, L=1 \mathrm{~m}$ & 188 & 0.37 & 0.93 \\
$a=1 \mathrm{~mm}, \Delta \phi_{3}= \pm 90^{\circ}, L=1 \mathrm{~m}$ & 188 & 0.08 & 1.56 \\
$a=1 \mathrm{~mm}, L=1 \mathrm{~m}$, first-third peaks, & 375 & 0.37 & 0.89 \\
& & & \\
\hline \hline
\end{tabular}

compression) is a method to swap the arrival order of the subpulses. The rotation of the global chirp may be also used to further reduce the time separation among the sub-pulses, accepting a smaller ratio of the current at the peaks and in between the maxima.

With the scheme we propose we can provide $3 \mathrm{kA}$ peak current two sub-bunches with a time delay tunable from 85 fs up to about 380 fs and an energy separation between about $0.6 \%$ and more than $1 \%$ assuming the constraint we have on the maximum energy gain in the last SwissFEL cavities. If we let the peak current of the subpulses to vary in a range of some kA we can further enlarge the time and the energy separation equivalent to how it is done in the twin-bunch configuration.

We optimized the geometry of the corrugated plates and its aperture to have these energy and time separations among the subpulses. Smaller or larger separations for the reference geometry may be obtained modifying the corrugation of the device.

\section{CONCLUSIONS}

We have presented a scheme based on wakefield generation to obtain a two-color operation mode in FEL facilities. We have shown that we can generate two subbunches independently tunable in time and separated in energy keeping constant the peak current. Compared to previous methods our approach does not degrade the lasing power, because the bunches lase individually to saturation, and does not significantly modify the machine settings in the low energy section, so that its implementation in the accelerator is simplified compared to the twin-bunches scheme. By changing the ratio of the wavelength of the wakefield source and the initial beam length this scheme may also produce multicolor bunches, also if with more limited tunability in time and energy. The same scheme could in principle be applied to any FEL or in the framework of plasma wakefield acceleration (PWFA).

\section{ACKNOWLEDGMENTS}

We would like to acknowledge T. Schietinger, H. H. Braun, and T. Garvey for the careful proofreading of the paper, and K. L. Bane for the fruitful discussions.

[1] R. W. Lee et al., Plasma-based studies with intense $X$-ray and particle beam sources, Laser Part. Beams 20, 527 (2002).

[2] A. Forsman, A. Ng, G. Chiu, and R. M. More, Interaction of femtosecond laser pulses with ultrathin foils, Phys. Rev. E 58, R1248 (1998).

[3] W. A. Hendrickson and C. M. Ogata, Phase determination from multiwavelength anomalous diffraction measurements, Methods Enzymol. 276, 494 (1997).

[4] H. N. Chapman et al., Femtosecond $X$-ray protein nanocrystallography, Nature (London) 470, 73 (2011).

[5] R. Bonifacio, C. Pellegrini, and L. Narducci, Collective instabilities and high-gain regime in a free electron laser, Opt. Commun. 50, 373 (1984).

[6] A. A. Lutman, R. Coffee, Y. Ding, Z. Huang, J. Krzywinski, T. Maxwell, M. Messerschmidt, and H.-D. Nuhn, Experimental Demonstration of Femtosecond Two-Color $X$-Ray Free-Electron Lasers, Phys. Rev. Lett. 110, 134801 (2013).

[7] T. Hara et al., Two-colour hard $X$-ray free-electron laser with wide tunability, Nat. Commun. 4, 2919 (2013).

[8] V. Petrillo et al., Observation of Time-Domain Modulation of Free-Electron-Laser Pulses by Multipeaked ElectronEnergy Spectrum, Phys. Rev. Lett. 111, 114802 (2013).

[9] A. Marinelli, A. A. Lutman, J. Wu, Y. Ding, J. Krzywinski, H.-D. Nuhn, Y. Feng, R. N. Coffee, and C. Pellegrini, Multicolor Operation and Spectral Control in a GainModulated X-Ray Free-Electron Laser, Phys. Rev. Lett. 111, 134801 (2013).

[10] P. Musumeci, R. K. Li, and A. Marinelli, Nonlinear Longitudinal Space Charge Oscillations in Relativistic Electron Beams, Phys. Rev. Lett. 106, 184801 (2011).

[11] M. Ferrario et al., Laser comb with velocity bunching: Preliminary results at SPARC, Nucl. Instrum. Methods Phys. Res., Sect. A 637, S43 (2011).

[12] M. J. Hogan et al., Plasma wakefield acceleration experiments at FACET, New J. Phys. 12, 055030 (2010).

[13] Z. Zhang, Y. Ding, A. Marinelli, and Z. Huang, Longitudinal dynamics of twin electron bunches in the Linac coherent light source, Phys. Rev. ST Accel. Beams 18, 030702 (2015).

[14] P. Emma et al., Experimental Demonstration of EnergyChirp Control in Relativistic Electron Bunches Using a Corrugated Pipe, Phys. Rev. Lett. 112, 034801 (2014).

[15] S. Bettoni, P. Craievich, M. Pedrozzi, and S. Reiche, Simulations of a corrugated beam pipe for the chirp compensation in SwissFEL, in Proceedings of International Free Electron Laser Conference (FEL'13), New York, 2013, p. 214.

[16] P. Craievich, Passive longitudinal phase space linearizer, Phys. Rev. ST Accel. Beams 13, 034401 (2010).

[17] F. Fu et al., Demonstration of Nonlinear-Energy-Spread Compensation in Relativistic Electron Bunches with Corrugated Structures, Phys. Rev. Lett. 114, 114801 (2015). 
[18] S. Antipov, S. Baturin, C. Jing, M. Fedurin, A. Kanareykin, C. Swinson, P. Schoessow, W. Gai, and A. Zholents, Experimental Demonstration of Energy-Chirp Compensation by a Tunable Dielectric-Based Structure, Phys. Rev. Lett. 112, 114801 (2014).

[19] S. Antipov, C. Jing, M. Fedurin, W. Gai, A. Kanareykin, K. Kusche, P. Schoessow, V. Yakimenko, and A. Zholents, Experimental Observation of Energy Modulation in Electron Beams Passing through Terahertz Dielectric Wakefield Structures, Phys. Rev. Lett. 108, 144801 (2012).

[20] S. Bettoni, P. Craievich, A. A. Lutman, and M. Pedrozzi, Temporal profile measurements of relativistic electron bunch based on wakefield generation, Phys. Rev. Accel. Beams 19, 021304 (2016).

[21] E. Allaria et al., Two-colour pump-probe experiments with a twin-pulse-seed extreme ultraviolet free-electron laser, Nat. Commun. 4, 2476 (2013).

[22] E. Ferrari et al., Widely tunable two-colour seeded freeelectron laser source for resonant-pump resonant-probe magnetic scattering, Nat. Commun. 7, 10343 (2015).

[23] A. Lutman et al., Demonstration of Single-Crystal SelfSeeded Two-Color $X$-Ray Free-Electron Lasers, Phys. Rev. Lett. 113, 254801 (2014).

[24] R. Ganter, SwissFEL conceptual design report, PSI Report No. 10-04, 2012.

[25] S. Bettoni, B. Beutner, and V. A. Goryashko, Microbunching instability studies in SwissFEL, in Proceedings of the 3rd International Particle Accelerator Conference, New Orleans, LA, 2012 (IEEE, Piscataway, NJ, 2012), p. 1744.

[26] K. L. F. Bane and G. Stupakov, Impedance of a rectangular beam tube with small corrugations, Phys. Rev. ST Accel. Beams 6, 024401 (2003).

[27] K. L. F. Bane and G. Stupakov, Report No. SLAC-PUB14925, 2013.

[28] M. Borland, ANL Advanced Photon Source Report No. LS-287, 2000.

[29] K. Bane and P. Emma, LiTrack: A fast longitudinal phase space tracking code with graphical user interface, in Proceedings of the 21st Particle Accelerator Conference, Knoxville, TN, 2005 (IEEE, Piscataway, NJ, 2005), p. 4266.

[30] S. Reiche, GENESIS 1.3: A fully 3D time-dependent FEL simulation code, Nucl. Instrum. Methods Phys. Res., Sect. A 429, 243 (1999).

[31] Z. Zhang, K. Bane, Y. Ding, Z. Huang, R. Iverson, T. Maxwell, G. Stupakov, and L. Wang, Electron beam energy chirp control with a rectangular corrugated structure at the Linac coherent light source, Phys. Rev. ST Accel. Beams 18, 010702 (2015).

[32] P. Emma, K. Bane, M. Cornacchia, Z. Huang, H. Schlarb, G. Stupakov, and D. Walz, Femtosecond and Subfemtosecond $X$-Ray Pulses from a Self-Amplified SpontaneousEmission-Based Free-Electron Laser, Phys. Rev. Lett. 92, 074801 (2004). 